\title{
A TERAPIA NUTRICIONAL NOS CUIDADOS PALIATIVOS
}

\section{ARTIGO DE REVISÃO}

CARDOSO, Camila Castro ${ }^{1}$

CARDOSO, Camila Castro. A terapia nutricional nos cuidados paliativos. Revista Científica Multidisciplinar Núcleo do Conhecimento. Ano. 07, Ed. 01, Vol. 03, pp. 153163. Janeiro de 2022. ISSN: 2448-0959, Link de acesso: https://www.nucleodoconhecimento.com.br/nutricao/terapia-nutricional, $\quad$ DOI: 10.32749/nucleodoconhecimento.com.br/nutricao/terapia-nutricional

\section{RESUMO}

Os pacientes em cuidados paliativos apresentam um estado de desnutrição agudo devido ao hipercatabolismo ocasionado pela situação de enfermidade em que se encontram. Neste contexto, o presente artigo disserta sobre 0 uso da terapia nutricional em pacientes no fim da vida, tendo como questão norteadora: o tratamento nutricional pode ser considerado válido e benéfico para a qualidade de vida dos pacientes em cuidados paliativos mesmo que para isso sejam utilizados métodos invasivos e incômodos ao paciente? Portanto, tem como objetivo responder a esta questão referente à aplicação da terapia nutricional em pacientes paliativos. Trata-se de uma revisão narrativa, utilizando as bases de dados Lilacs e Scielo. Como resultado, constatou-se que a terapia nutricional, em alguns casos, parece contribuir para a diminuição da desnutrição, podendo desencadear, até mesmo, algumas outras melhorias no quadro evolutivo do paciente, como o aumento do sistema imunológico. No entanto, para uma parcela de profissionais da saúde, esses benefícios são questionáveis diante das consequências e complicações que esse suporte nutricional pode causar aos pacientes críticos. Devido à escassez de evidências científicas para a decisão da administração da terapia nutricional e pela alimentação ter um forte

\footnotetext{
${ }^{1}$ Pós-graduação em Nutrição vegetariana, Pós-Graduação em Cuidados Paliativos e Terapia da Dor, Graduação em Nutrição. ORCID: 0000-0002-6838-6953.
}

RC: 105095

Disponível em: https://www.nucleodoconhecimento.com.br/nutricao/terapianutricional 
significado cultural, concluiu-se que esta decisão deverá ser multiprofissional e ter a aceitação da família por escrito, caso o paciente esteja impossibilitado de decidir. Caso o paciente opte por não receber o apoio nutricional, sua decisão deverá ser respeitada e atendida pela equipe e por seus familiares, valorizando sua autonomia.

Palavras-chave: terapia nutricional, cuidados paliativos, qualidade de vida.

\section{INTRODUÇÃO}

Segundo Morais (2016), em 2002 a Organização Mundial de Saúde (OMS) atualizou o conceito de cuidados paliativos (CP) definido em 1990. Essa atualização aceita internacionalmente entende que:

Cuidado Paliativo é a abordagem que promove qualidade de vida dos pacientes e familiares que enfrentam problemas associados a doenças ameaçadoras de vida por meio da prevenção e alívio do sofrimento, por meios de identificação precoce, avaliação e tratamento da dor e outros problemas de natureza física, psicossocial e espiritual.

Esses cuidados também podem ser indicados as pessoas que receberam tratamento curativo e aos sobreviventes do câncer que tiveram efeitos adversos tardios ou comorbidades relacionadas aos tratamentos recebidos.

Ainda segundo Morais (2016), a OMS entende a qualidade de vida como a percepção do indivíduo em relação a sua posição na vida, no contexto da cultura e do sistema de valores em que vive, considerando seus objetivos, expectativas, padrões e interesses. A partir disso, percebe-se ser possível minimizar o sofrimento, levando em consideração os cuidados ativos.

Em relação à nutrição e à alimentação em $\mathrm{CP}$, estes apresentem papel preventivo, pois possuem meios e vias de alimentação que reduzem os efeitos adversos provocados pelos tratamentos, como: o retardamento da síndrome de anorexiacaquexia, a ressignificação do alimento e o auxílio no controle de sintomas, mantendo a hidratação controlada, preservando o peso e a composição corporal satisfatória. 
Como aponta Reiriz (2008), o suporte nutricional, para uma parcela da comunidade científica, nestes casos de terminalidade, beneficia o paciente. Não obstante, há uma parte cada vez maior de profissionais da área paliativista, que questiona os verdadeiros benefícios deste suporte nutricional a estes pacientes.

Benarroz (2009) lembra que nesses assuntos pertinentes à alimentação, é normal existirem dilemas bioéticos, pois o ato de se alimentar está relacionado ao estilo de vida e ao bem-estar, bem como a valores culturais, sociais, relações familiares, sendo, ainda, um símbolo cultural de vitalidade. Por isso, Reiriz (2008) recorda que esses profissionais alegam que o desconforto e as complicações advindas da terapia nutricional (TN) se sobrepõem aos seus benefícios para a qualidade de vida dos pacientes que não possuem possibilidade de cura.

No período final, alguns pacientes podem ter consequências como o desconforto abdominal e náuseas devido à alimentação oral que estes mantêm para agradar aos seus familiares. Nessa fase final da vida, a alimentação ainda é um assunto polêmico e que envolve contradições, mitos e emoções; por isso, é importante que as condutas sejam definidas de acordo com o desejo do paciente e da família (BENARROZ, 2009).

Nesse contexto, Morais (2016) relata que o primordial no CP é preservar a dignidade do paciente e proporcionar conforto e bem-estar. Embora os CP devam respeitar os anseios do paciente, fornecer o maior conforto possível por meio das terapias indicadas e favorecer a redução do sofrimento, há contestações se a alimentação pode ou não contribuir com esse processo.

Sendo assim, levantou-se o seguinte questionamento: o tratamento nutricional pode ser considerado válido e benéfico para a qualidade de vida dos pacientes em cuidados paliativos mesmo que para isso sejam utilizados métodos invasivos e incômodos ao paciente? Portanto, tem-se como objetivo responder a esta questão referente a aplicação da terapia nutricional em pacientes paliativos. Como metodologia, adotouse a revisão narrativa, utilizando as bases de dados Lilacs e Scielo. As palavras-chave utilizadas na busca dos materiais foram: terapia nutricional, cuidados paliativos, 
qualidade de vida. Após as pesquisas, foram coletados oito trabalhos, sendo aplicados os critérios de inclusão que versavam sobre o contexto do trabalho e a proximidade destas palavras com o assunto, restando três artigos selecionados devido ao conteúdo, a argumentação e aos resultados apresentados. Os demais foram excluídos, pois, apesar de conterem as palavras-chave, o conteúdo não estava alinhado ao assunto pesquisado.

\section{ENTENDENDO O PACIENTE PALIATIVO}

De acordo com Reiriz (2008), o paciente terminal é entendido como aquele que possui uma enfermidade avançada, progressiva e sem possibilidade de cura, apresentando uma piora no quadro clínico recente, incluindo consequências como: anorexia, emagrecimento, xerostomia, desconforto respiratório, um declínio no aspecto comportamental, dificuldade de locomoção, higiene, continência, entre outros. Esses sintomas geram impacto emocional no paciente e em sua família. Para esse tipo de paciente deve-se sempre ter em mente o grau de necessidade de um suporte nutricional e se este suporte não irá acarretar desconfortos para o doente; não ignorando o princípio ético da não-maleficência.

Reiriz (2008) declara, também, que os pacientes em estado crítico apresentam características como o hirpermetabolismo e catabolismo, o que causa uma rápida perda de peso e desnutrição. Sendo a terapia nutricional um procedimento comum em tais pacientes e alguns dos seus benefícios são: a diminuição da resposta catabólica, o aumento da imunidade, a melhora tanto estrutural como funcional do trato gastrointestinal, a diminuição de complicações e tempo hospitalar.

Benarroz (2009) cita que a doença cancerígena tem um significativo impacto na capacidade física, no bem-estar psicológico e na vida social dos pacientes, apresentando uma forte consequência no estado nutricional e na qualidade de vida deles. Portanto, a má alimentação é normal em pacientes oncológicos. Quando a doença já no estado avançado, há sintomas e sinais como: náusea, vômito, alteração do paladar, saciedade precoce, anorexia, caquexia, fraqueza, dispneia, mudanças 
metabólicas, mucosite, diminuição da absorção de calorias; o que leva a uma diminuição da ingesta de alimentos e consequentemente perda de peso, resultando em efeitos indesejados no quadro clínico do paciente, como o agravo da doença e uma qualidade de vida prejudicada.

A xerostomia (sensação de boca seca) e a disgeusia (alteração do paladar), são sintomas recorrentes em pacientes que fazem tratamento com opioides, quimioterapia e radioterapia e são sintomas frequentes de desnutrição. A xerostomia tem sérias implicações como: dificuldade maior para deglutir alimentos, infecções da mucosa bucal, cáries, alterações de sono e alteração do paladar. Os problemas relacionados à saúde bucal limitam as opções de escolha e consumo dos alimentos, por isso há um forte impacto no estado nutricional do paciente (BENARROZ, 2009).

Como aponta Reiriz (2008), outra queixa comum é a perda de peso, que pode estar ligada à ingestão inadequada de alimentos e à desnutrição. Cerca de $80 \%$ dos pacientes com câncer avançado passam pela experiência da caquexia, que pode ser caracterizada, além da perda de peso, por lipólise, fraqueza muscular, anorexia, náuseas crônicas e diminuição da força física. Essa síndrome, comumente, é vivenciada por pacientes terminais e marca o avanço da doença, sendo reportada como o maior contribuinte da causa de óbito desses pacientes. Nesse contexto, Benarroz (2009), relata que no momento que o enfermo nota as mudanças na sua autoimagem, ocorre um grande impacto negativo, pois estas simbolizam para ele a aproximação da morte, a perda da sua autonomia, a fragilidade física e psicológica, refletindo diretamente na sua qualidade de vida. Reiriz (2008) afirma que um terço dos pacientes vão a óbito por conta da caquexia causada pelo tumor, mesmo após a remoção do mesmo através de cirurgia.

A suplementação parenteral em pacientes caquéticos tem sido usada e, em algumas situações, mostrou-se eficiente, interrompendo algumas sequelas metabólicas da doença. No entanto, estudos randomizados controlados mostram que a terapia nutricional agressiva não tem impacto positivo na resposta tumoral ou na sobrevida. 
Portanto, a nutrição via oral ainda é a opção ideal para pacientes que estão em CP (REIRIZ, 2008).

\section{TERAPIA NUTRICIONAL E SEUS MÉTODOS}

Conforme Reiriz (2008) a NHA (nutrição e hidratação artificial) é normalmente administrada por via enteral através de um tubo nasogástrico ou por gastrostomia/jejunostomia. A terapia nutricional enteral (TNE) é preferencial para administração de alimentos. A terapia nutricional parenteral, também, é considerada uma via para a realização do suporte nutricional através de acesso venoso central ou periférico. Esse tipo de nutrição, tem sido associado com: atrofia da mucosa intestinal, hiperglicemia, efeitos adversos na função imune, aumento do risco de complicações infecciosas e aumento da mortalidade em pacientes com doenças graves. Já a nutrição parenteral total (NPT), deve ser reservada para pacientes em que o trato gastrointestinal permanece indisponível por um período de tempo prolongado.

Morais (2016) aponta que as necessidades nutricionais de calorias, proteínas e hidratação, devem ser pautadas conforme a aceitação, tolerância e sintomas do paciente, visando à promoção do conforto e proporcionando melhor qualidade de vida, garantindo, não apenas, a ingestão adequada desses nutrientes, mas, evitando, em alguns casos, intervenções nutricionais invasivas, como a introdução de TNE ou NPT. Para isso, é necessário realizar uma avaliação que possibilite identificar os pacientes que possam se beneficiar com tais suportes nutricionais.

Nas palavras de Reiriz (2008), a NPT tem morbidade de 15\%, além de ter alto custo, sendo indicada, geralmente, para pacientes cirúrgicos com tumor gastrointestinal desnutridos e para pacientes que fazem tratamento com altas doses de quimioterapia para transplante de medula óssea. Já a nutrição enteral (TNE), deve ser avaliada em pacientes no qual o intestino funcione, porém não conseguem ter ingestão adequada por via oral, por exemplo, em pacientes com neoplasia de cabeça e pescoço ou com carcinoma esofágico. Em estudos não-controlados com pacientes portadores de neoplasia de cabeça e pescoço, realizando modalidade terapêutica combinada, o 
suporte nutricional foi associado ao aumento do peso corporal e melhora da qualidade de vida.

\section{A INTERAÇÃO ALIMENTAÇÃO-PACIENTE}

O estudo de Silva et al. (2010 apud MORAIS, 2016), aponta que as intervenções nutricionais e médicas refletem indiretamente na socialização e possibilitam a sua participação nas refeições junto aos familiares e amigos, além de proporcionar conforto e autocuidado. Isso mostrou que os aspectos nutricionais do paciente não são apenas aqueles relacionados ao estado nutricional, mas também a relação familiar, sensação de prazer, bem-estar e autonomia.

Segundo Seredynskyj et al. (2014 apud MORAIS, 2016), a autonomia deve ser mantida para a manutenção de um melhor estado de saúde do enfermo. Junto a isso, o tratamento médico e nutricional acarretou, também, a redução dos sintomas, como: inapetência, disgeusia, candidíase oral, mucosite, náuseas e constipação (DURVAL et al., 2010 apud MORAIS, 2016).

Em um outro estudo apresentado por Hasenberg et al. (2010 apud MORAIS, 2016) verificou-se que a terapia nutricional precoce pode manter a composição corporal, melhorar a condição de vida e, ainda, prolongar a sobrevida de pacientes oncológicos que estão em CP. Este, também, avaliou os efeitos da NPT e da nutrição oral. O grupo que recebeu nutrição parenteral e oral obteve nível de albumina estável, além de reduzir sintomas gastrintestinais relacionados a quimioterapia, implicando na melhora da qualidade de vida. Os pacientes que receberam somente a suplementação via oral apresentaram melhora dos sintomas como saciedade precoce, constipação, náuseas, vômitos, dor abdominal e diarreia, resultando em aumento significativo do apetite. Logo, a intervenção nutricional atuou de forma positiva nos aspectos clínicos e sociais, melhorando a condição de vida desses pacientes estudados.

Por outro lado, Morais (2016) relata que o monitoramento do peso, para grande parte dos pacientes, pode ser prejudicial ou sem utilidade, pois a redução desse mostra-se 
assustadora e estressante, já que essa situação insinua a aproximação do processo da morte.

Reiriz (2008) lembra que mesmo que se tenha o conhecimento do quanto o suporte nutricional é considerado um componente fundamental nos cuidados de um paciente crítico, acredita-se que a terapia nutricional não está livre de riscos e efeitos colaterais, sendo um procedimento muitas vezes fútil e indesejado pelos pacientes. É importante salientar que essa terapia, quando utilizada, deve ser administrada de forma segura e eficaz, na presença de uma equipe multidisciplinar e, como resultado, apresentar a redução de deficiências nutricionais, complicações e custos.

Afirma, também, que a nutrição artificial está associada a riscos consideráveis. Por exemplo, os pacientes com demência avançada, que recebem a alimentação via gastrostomia, têm maior risco de aspiração e, consequentemente, a pneumonia, a diarreia, ao desconforto gastrointestinal, além de problemas associados a remoção do tubo alimentar. Nessa fase da doença, orienta-se ter como alvo o conforto do paciente, não devendo fazer uso desse tipo de terapia. É importante lembrar que a nutrição e a hidratação artificiais podem causar desconforto no processo do falecimento (REIRIZ, 2008).

Apesar de apresentar alguns bons resultados como os citados acima, deve-se ter em mente os desconfortos que o paciente pode ser submetido ao realizar a terapia nutricional. Diante desses aspectos, entende-se que a abordagem nutricional deve, primordialmente, oferecer conforto e prazer sem deixar de respeitar a autonomia do paciente e de seus familiares. Sendo assim, quando associada com outras medidas terapêuticas, poderá contribuir para a promoção da qualidade de vida dos pacientes em fase terminal (MORAIS, 2016). 


\section{O USO DA TERAPIA NUTRICIONAL}

Benarroz (2009) declara que a decisão de fornecer ou não o suporte nutricional para pacientes em cuidados paliativos demanda do conhecimento da vontade do paciente, das expectativas dele e de sua família, diante de uma conversa aberta e clara.

Reiriz (2008) aponta que, nos últimos vinte anos, profissionais de saúde tentam chegar a um consenso sobre o uso adequado da terapia nutricional artificial, sendo o suporte nutricional considerado um tratamento que os pacientes e seus familiares podem aceitar ou recusar baseados nos benefícios, nos riscos inerentes ao tratamento, no desconforto que pode causar ao paciente, na crença religiosa e cultural. Essa decisão é encarada como um dilema ético que deve levar em consideração os princípios de autonomia, beneficência, não-maleficência e justiça. Para aqueles que optarem por não receber o tratamento, deve ser fornecido cuidados paliativos compreensivos, aliviando os sintomas físicos e psicológicos, incluindo suporte emocional e espiritual. A decisão da recusa aos tratamentos e terapias que sustentam a vida em condições específicas não é somente técnica, tendo um forte embasamento moral envolvido, pois a alimentação tem um valor simbólico significativo na sociedade. Levando em conta os conflitos culturais que tais debates geram, é primordial ter um olhar individualizado sobre as diferentes demandas clínicas que cada paciente exige.

Conforme Benarroz (2009), ainda que o objetivo dos cuidados paliativos seja oferecer uma rede de apoio para que os pacientes e familiares possam passar por esta fase da melhor maneira possível, em algumas situações, os profissionais de saúde, se encontram em conflitos bioéticos, principalmente ao enfrentar a impossibilidade de alimentação ou hidratação, cuidados considerados básicos.

Ainda segundo o autor supracitado, a alimentação representa saúde e bem-estar, e é considerada essencial para a sobrevivência. A sua privação é entendida como ir contra a vida, portanto, para alguns pacientes, não conseguir se alimentar ou não poder é, em muitos casos, entendido como a piora da saúde. Reiriz (2008) afirma que 
os Guidelines da Academia Americana de Neurologia e da Associação Médica Americana, permitem que médicos deem continuidade ou cessem a terapia nutricional de pacientes neurologicamente prejudicados quando tal ato foi determinado pelo paciente antes da fase aguda ou por seus cuidadores.

Por fim, Reiriz (2008) relata que, apesar de se saber que a terapia nutricional pode trazer danos quando administrada em pacientes terminais ou que não apresenta algum benefício para estes, muitas pessoas acreditam que é mais tolerável dar continuidade ao tratamento do que retirá-lo, crendo que a atitude de cessar o tratamento, levará o paciente à morte. Contudo, a escassez da alimentação não será o que irá acarretar a morte do paciente, mas sim a enfermidade de base que atua como elemento determinante.

\section{CONCLUSÃO}

Optar entre manter ou retirar a terapia nutricional é uma decisão difícil e conflitante, principalmente para os profissionais que estão acompanhando o paciente. Muitos acreditam que o objetivo primordial é a preservação ou restauração da vida, no entanto, a equipe, deve voltar sua atenção ao conforto do paciente, incluindo alívio de sintomas como: dispneia, náuseas, fadiga e depressão. Em muitos casos, o alimento nem sempre irá promover o conforto e o bem-estar que se espera, as consequências indesejáveis da alimentação artificial em especial, são em algumas situações, motivo da queda da qualidade de vida, prejudicando o verdadeiro objetivo dos cuidados no fim da vida (REIRIZ, 2008).

Benarroz (2008) afirma que a visão filosófica dos cuidados paliativos está baseada nos princípios de valorização da vida e consideração da morte como um processo natural, sem abreviar e prolongar a vida. Porém, a aplicação na prática é desafiadora e demanda uma equipe interdisciplinar adequada e treinada ao atendimento humanizado. 
Diante do exposto e retomando à questão norteadora sobre o tratamento nutricional ser considerado válido e benéfico para a qualidade de vida dos pacientes em cuidados paliativos mesmo que para isso sejam utilizados métodos invasivos e incômodos ao paciente, concluiu-se que alguns estudos apresentam bons resultados no uso da terapia nutricional para estes pacientes, podendo sim melhorar a qualidade de vida deles, sendo benéfica ao paciente. Mas, por outro lado, como relatado, esta mesma terapia pode causar problemas e desconforto ao enfermo. Logo, vale a reflexão no momento da decisão, levando em conta o quanto a terapia irá ser benéfica à qualidade de vida e ao bem-estar do paciente, sem deixar de lado o valor primordial dos cuidados paliativos que é o conforto do paciente e sua autonomia. Portanto, os profissionais integrados à equipe decidirão junto paciente e familiares qual será a melhor conduta a ser aplicada.

Reiriz (2008) declara que, devido ao baixo número existente de evidências cientificas para esta decisão e pelo motivo da alimentação de ter uma importante influência cultural, a escolha da terapia nutricional até a morte, deve ser multiprofissional e ter a concordância por escrito da família, caso o paciente não tenha condições de decidir. Nos casos em que o paciente escolha por não receber mais o tratamento nutricional, a sua decisão deve ser respeitada e consentida pela equipe de saúde e por seus cuidadores, pois acima de qualquer resultado científico está o princípio da autonomia do paciente, assim como os da beneficência e não-maleficência. Neste contexto, observa-se a necessidade de mais estudos de nutrição envolvendo pacientes na fase terminal da vida, não somente oncológicos, mas também nas várias situações que englobam esse assunto.

\section{REFERÊNCIAS}

BENARROZ, Monica de Oleveira; FAILLACE, Giovanna Borges Damião; BARBOSA, Leandro Augusto. Bioética e nutrição em cuidados paliativos oncológicos em adultos. Caderno de Saúde Pública, Rio de Janeiro, v. 9, n. 25, p. 1875-1882, set. 2009. Disponível

em: 
https://www.scielo.br/j/csp/a/Ps5LcthbYh4qmxwQGJtHR3J/abstract/?lang=pt. Acesso em 04/01/2022

MORAIS, Suelyne Rodrigues de; BEZERRA, Alane Nogueira; CARVALHO, Natália Sales de; VIANA, Ana Carolina Cavalcante. Nutrição, qualidade de vida e cuidados paliativos: uma revisão integrativa. Revista Dor, São Paulo, v. 17, n. 2, p. 136-140, abr. $2016 . \quad$ Disponível em: https://www.scielo.br/j/rdor/a/3Vtn74msRR94xyhgmWTQg5w/?lang=pt. Acesso em 06/12/2021.

REIRIZ, André Borba; MOTTER, Carine; BUFFON, Viviane Raquel; SCATOLA, Ronei Pacheco; FAY, Alexandre Schio; MANZINI, Michelle. Cuidados paliativos - há benefícios na nutrição do paciente em fase terminal? Revista da Sociedade Brasileira de Clínica Médica, Caxias do Sul, v. 4, n. 6, p. 150-155, 17 jun. 2008. Disponível em: http://bases.bireme.br/cgi-

bin/wxislind.exe/iah/online/? IsisScript=iah/iah.xis\&src=google\&base=LILACS\&lang=p \&nextAction=Ink\&exprSearch=494996\&indexSearch=ID Acesso em 04/01/2022

Enviado: Outubro, 2021.

Aprovado: Janeiro, 2022. 